\title{
Article \\ Positive Solutions for a Class of Nonlinear Singular Fractional Differential Systems with Riemann-Stieltjes Coupled Integral Boundary Value Conditions ${ }^{\dagger}$
}

\author{
Daliang Zhao ${ }^{1}$ and Juan Mao ${ }^{2, *}$ \\ 1 School of Mathematics and Statistics, Shandong Normal University, Jinan 250014, China; \\ dlzhao928@sdnu.edu.cn \\ 2 Department of Basic Courses, Shandong Polytechnic, Jinan 250104, China \\ * Correspondence: maojuan@sdp.edu.cn or jmao_sp@163.com \\ + Research supported by the project of Shandong Province Higher Educational Science and Technology \\ Program of China under the grant J18KA233, the Natural Science Fund for Distinguished Young Scholars of \\ Shandong Province under grant JQ201613.
}

Citation: Zhao, D.; Mao, J. Positive Solutions for a Class of Nonlinear Singular Fractional Differential Systems with Riemann-Stieltjes Coupled Integral Boundary Value Conditions. Symmetry 2021, 13, 107. https://doi.org/10.3390/sym13010107

Received: 10 December 2020 Accepted: 6 January 2020 Published: 8 January 2021

Publisher's Note: MDPI stays neutral with regard to jurisdictional clai$\mathrm{ms}$ in published maps and institutional affiliations.

Copyright: (C) 2021 by the authors. Licensee MDPI, Basel, Switzerland. This article is an open access article distributed under the terms and conditions of the Creative Commons Attribution (CC BY) license (https:// creativecommons.org/licenses/by/ $4.0 /)$.

\begin{abstract}
In this paper, sufficient conditions ensuring existence and multiplicity of positive solutions for a class of nonlinear singular fractional differential systems are derived with Riemann-Stieltjes coupled integral boundary value conditions in Banach Spaces. Nonlinear functions $f(t, u, v)$ and $g(t, u, v)$ in the considered systems are allowed to be singular at every variable. The boundary conditions here are coupled forms with Riemann-Stieltjes integrals. In order to overcome the difficulties arising from the singularity, a suitable cone is constructed through the properties of Green's functions associated with the systems. The main tool used in the present paper is the fixed point theorem on cone. Lastly, an example is offered to show the effectiveness of our obtained new results.
\end{abstract}

Keywords: fractional differential equations; singularity; coupled integral boundary value conditions; cone; fixed point theorem

\section{Introduction}

Recently, differential equations of fractional order have received more and more attention in virtue of their various applications in the fields of science and engineering. Compared with integer-order differential equations, fractional-order models can provide more accurate characterizations of many natural phenomena and mathematical problems. Many good results about fractional differential equations have been obtained in some recent literature. For more details, one can see [1-17] and references therein. On the other side, the coupled problem of differential equations is one of the hot topics in applied mathematics, biology, biomathematics, and other fields. As is well known, coupled conditions in reaction-diffusion equations can reflect the interactions between diffusion and reaction to a great extent. For instance, the prey-predator reaction-diffusion system, which is one of the types of coupled models, can describe the relationships between prey density and predator density through some specific time and location variables. See [18-21] for a good overview.

Some existing papers [22-29] have studied the positive solutions for various boundary value problems by using different methods of nonlinear functional analysis. For example, Asif et al. [24] considered a integral (second)-order differential system with coupled boundary value conditions. They found the existence of positive solutions by Krasnoselskii's fixed point theorem with the singularity only at $t=0$ and $t=1$. Y. Cui et al. [25] also studied a class of second-order differential equations involving coupled integral boundary value conditions, and they derived the existence and uniqueness result by a mixed monotone method. The singularity in this paper was allowed at $t=0,1$ and at only one of 
the variables within the nonlinear items. C. Yuan et al. [27] obtained some results about multiple positive solutions of a nonsingular fractional differential equation with coupled boundary value conditions in view of some fixed point theorems on cone. W. Yang [23] and D. Zhao et al. [28], by utilizing a nonlinear alternative of the Leray-Schauder type and Krasnoselskii's fixed point theorem together with the theory of fixed point index on cone, derived some existence results of multiple positive solutions for some fractional semipositone boundary value problems with coupled integral boundary value conditions. However, the limitation was that the nonlinear functions here were all not provided with singularity.

Inspired by the above works, we consider the following nonlinear fractional singular systems with coupled integral boundary value conditions involving Riemann-Stieltjes integrals in Banach spaces:

$$
\left\{\begin{array}{l}
D_{0^{+}}^{\alpha} u(t)+f(t, u(t), v(t))=0, \quad t \in(0,1), \\
D_{0^{+}}^{\alpha} v(t)+g(t, u(t), v(t))=0, \quad t \in(0,1), \\
u^{(j)}(0)=0, j=0,1, \\
v^{(j)}(0)=0, j=0,1, \\
u^{\prime}(1)=\int_{0}^{1} v(t) d \phi(t), v^{\prime}(1)=\int_{0}^{1} u(t) d \psi(t),
\end{array}\right.
$$

where $D_{0^{+}}^{\alpha}$ denotes the Riemann-Liouville fractional derivative of order $\alpha \in(2,3] ; f, g \in$ $C[(0,1) \times(0,+\infty) \times(0,+\infty),[0,+\infty)]$, that is, $f(t, u, v), g(t, u, v)$ may be singular at $t=0$, $t=1$ and $u=0, v=0 ; \int_{0}^{1} u(t) d \psi(t)$ and $\int_{0}^{1} v(t) d \phi(t)$ denote the Riemann-Stieltjes integrals of $u$ and $v$ with respect to $\psi$ and $\phi$, respectively, where $\psi$ and $\phi$ are right continuous on $[0,1)$, left continuous at $t=1$, and nondecreasing on $[0,1]$ with $\phi(0)=\psi(0)=0$. To the best of our knowledge, no contributions exist investigating the existence and multiplicity of positive solutions for such fractional singular system with coupled integral boundary value conditions.

We point out that there are several basic methods to deal with the singularity occurring at both $t=0,1$ and $u$ or $v=0$. For example, one is a sequential-based method and the other is a mixed monotone method. The main function of the first method is to transform the singular system into an approximate system by using sequential techniques. Some superlinear or sublinear conditions are usually supposed to the nonlinear functions. The mixed monotone method is used to solve the unique positive solution of some singular system, for which the variables of nonlinear terms should satisfy some monotonicity conditions. Unlike the above two methods, in this paper, the classical cone compression and expansion fixed point theorem, together with some necessary assumptions on the nonlinear functions in singular system (1), is utilized to overcome the singularity. This is based on the good properties of Green's function and a special cone corresponding to system (1). Relatively speaking, mixed monotone method has low demands on the properties of Green's function and the corresponding cone, and the sequential-based method is usually utilized in abstract spaces with many abstract space theories such as Arzelà-Ascoli theorem, noncompactness measure theory, etc. Therefore, for solving the singularity problem of the same kind, the method adopted in this paper is relatively concise and has its own advantages. Notably, this is also attributed to the assumptions of the nonlinear terms and the structure of the special singular system in the present paper.

The proposed singular fractional order coupled system (1) here, which generalizes the cases of singular integral (second)-order differential equations studied in [24,25] and [22], has more extensive and valid applications. The nonlinear items in this paper are allowed to be singular not only at time $t=0,1$, but also at both variables $u, v=0$ in contrast to the above-mentioned literature [24,25,27] and [29]. In addition, monotonicity with respect to variables $u, v$ in nonlinear items, which emerge in $[25,29]$, is not needed in this paper. By deriving Green's functions in the integral representation of system (1) and their important properties, we establish a special cone in an appropriate Banach space. 
This is an especially significant step for our subsequent proofs. Through utilizing cone theory and the classical cone compression and expansion fixed point theorem, sufficient conditions ensuring existence and multiplicity of positive solutions for system (1) are derived, respectively.

An outline of the present paper is as follows. Some necessary preliminaries are presented in Section 2. In Section 3, sufficient conditions of existence and multiplicity of positive solutions for the addressed systems are investigated. In Section 4, we present an example to illustrate the obtained new results.

\section{Preliminaries}

For convenience, we first list some results about fractional calculus in common use that can be found in the recent literature [30,31].

Definition 1. The Riemann-Liouville standard fractional integral of order $\alpha>0$ of a continuous function $u:(0,+\infty) \rightarrow \mathbb{R}$ is given by

$$
I_{0^{+}}^{\alpha} u(t)=\frac{1}{\Gamma(\alpha)} \int_{0}^{t}(t-s)^{\alpha-1} u(s) d s,
$$

provided that the right-side integral is pointwise defined on $(0,+\infty)$.

Definition 2. The Riemann-Liouville fractional derivative of order $\alpha$ of a continuous function $u:(0,+\infty) \rightarrow \mathbb{R}$ is defined by

$$
\left(D_{0+}^{\alpha} u\right)(t)=\frac{1}{\Gamma(n-\alpha)}\left(\frac{d}{d t}\right)^{n} \int_{0}^{t} \frac{u(s)}{(t-s)^{\alpha-n+1}} d s,
$$

where $n=[\alpha]+1$, provided that the right side is pointwise defined on $(0,+\infty)$.

Lemma 1. Let $\alpha>0$, then the differential equation

$$
D_{0^{+}}^{\alpha} u(t)=0
$$

has solutions $u(t)=c_{1} t^{\alpha-1}+c_{2} t^{\alpha-2}+\cdots+c_{N} t^{\alpha-N}$, for some $c_{i} \in \mathbb{R}, i=0,1,2, \cdots, N$, where $N$ is the smallest integer greater than or equal to $\alpha$.

Lemma 2. Assume that $u \in C(0,1) \cap L^{1}[0,1]$ with a fractional derivative of order $\alpha>0$ that belongs to $C(0,1) \cap L^{1}[0,1]$. Then

$$
I_{0^{+}}^{\alpha} D_{0^{+}}^{\alpha} u(t)=u(t)+c_{1} t^{\alpha-1}+c_{2} t^{\alpha-2}+\cdots+c_{N} t^{\alpha-N},
$$

for some $c_{i} \in \mathbb{R}, i=1,2, \cdots, N$.

Let space $E=C[0,1]$ be equipped with the maximum norm $\|u\|=\max \{|u(t)|: t \in$ $[0,1]\}$ for each $u \in E$. $(E,\|\cdot\|)$ is a Banach space. Similarly, for each $(u, v) \in E \times E$, we define $\|(u, v)\|_{1}=\max \{\|u\|,\|v\|\}$. It is not difficult to verify that $\left(E \times E,\|\cdot\|_{1}\right)$ is also a Banach space.

Now, for convenience, we present the assumptions as follows:

Hypothesis 1. $f, g \in C[(0,1) \times(0,+\infty) \times(0,+\infty),[0,+\infty)]$, and there exist constants $\lambda_{i}, \mu_{i}$ with $-\infty<\lambda_{i}<0<\mu_{i}<+\infty, i=1,2,3,4$, and constants $N, M>0$ with $0<N \leq 1 \leq M$, such that for all $t \in(0,1), u, v \in(0,+\infty)$,

$$
c_{1}^{\mu_{1}} c_{2}^{\mu_{2}} f(t, u, v) \leq f\left(t, c_{1} u, c_{2} v\right) \leq c_{1}^{\lambda_{1}} c_{2}^{\lambda_{2}} f(t, u, v), 0<c_{i} \leq N, i=1,2,
$$




$$
\begin{gathered}
c_{1}^{\lambda_{1}} c_{2}^{\lambda_{2}} f(t, u, v) \leq f\left(t, c_{1} u, c_{2} v\right) \leq c_{1}^{\mu_{1}} c_{2}^{\mu_{2}} f(t, u, v), c_{i} \geq M, i=1,2, \\
c_{1}^{\mu_{3}} c_{2}^{\mu_{4}} g(t, u, v) \leq g\left(t, c_{1} u, c_{2} v\right) \leq c_{1}^{\lambda_{3}} c_{2}^{\lambda_{4}} g(t, u, v), 0<c_{i} \leq N, i=1,2, \\
c_{1}^{\lambda_{3}} c_{2}^{\lambda_{4}} g(t, u, v) \leq g\left(t, c_{1} u, c_{2} v\right) \leq c_{1}^{\mu_{3}} c_{2}^{\mu_{4}} g(t, u, v), c_{i} \geq M, i=1,2 .
\end{gathered}
$$

Hypothesis 2. $f(\cdot, 1,1), g(\cdot, 1,1) \in C[(0,1),[0,+\infty)]$ and satisfy $0<\int_{0}^{1} t(1-t)^{\alpha-2} f(t, 1,1) d t$ $<+\infty, 0<\int_{0}^{1} t(1-t)^{\alpha-2} g(t, 1,1) d t<+\infty$.

Hypothesis 3. $\int_{0}^{1} t^{\alpha-1} d \phi(t) \cdot \int_{0}^{1} t^{\alpha-1} d \psi(t)<(\alpha-1)^{2}$. For the purpose of facilitating our next proofs, we give the following notations:

$$
\begin{gathered}
\omega_{1}=\int_{0}^{1} \frac{t^{\alpha-1}}{\alpha-1} d \phi(t), \omega_{2}=\int_{0}^{1} \frac{t^{\alpha-1}}{\alpha-1} d \psi(t), \\
\omega=1-\omega_{1} \omega_{2}, \omega=\min \left\{N, \frac{1}{M}\right\}, \\
\lambda_{0}=\min \left\{\lambda_{1}+\lambda_{2}, \lambda_{3}+\lambda_{4}\right\}, \lambda^{0}=\max \left\{\lambda_{1}+\lambda_{2}, \lambda_{3}+\lambda_{4}\right\}, \mu_{0}=\max \left\{\mu_{1}+\mu_{2}, \mu_{3}+\mu_{4}\right\} .
\end{gathered}
$$

Remark 1. (i) The representative functions satisfying the condition (Hypothesis 1) can be provided as follows:

$$
\begin{aligned}
f(t, u, v)= & \sum_{i=1}^{n} l_{i}(t) u^{\xi_{i}} v^{\eta_{i}}, \text { where } l_{i} \in C\left[(0,1), R^{+}\right], \text {and } \\
& \lambda_{1}=\xi_{1} \leq \xi_{2} \leq \cdots \leq \xi_{k}<0<\xi_{k+1} \leq \cdots \leq \xi_{n}=\mu_{1} ; \\
& \lambda_{2}=\eta_{1} \leq \eta_{2} \leq \cdots \leq \eta_{k}<0<\eta_{k+1} \leq \cdots \leq \eta_{n}=\mu_{2},
\end{aligned}
$$

for $i=1,2, \cdots, n ; k=1,2, \cdots, n-1$.

$g(t, u, v)=\sum_{i=1}^{n} k_{i}(t) u^{\zeta_{i} \vartheta^{\varsigma_{i}}}$, where $k_{i} \in C\left[(0,1), R^{+}\right]$, and

$$
\begin{aligned}
& \lambda_{3}=\zeta_{1} \leq \zeta_{2} \leq \cdots \leq \zeta_{k}<0<\zeta_{k+1} \leq \cdots \leq \zeta_{n}=\mu_{3} ; \\
& \lambda_{4}=\varsigma_{1} \leq \varsigma_{2} \leq \cdots \leq \varsigma_{k}<0<\varsigma_{k+1} \leq \cdots \leq \varsigma_{n}=\mu_{4},
\end{aligned}
$$

for $i=1,2, \cdots, n ; k=1,2, \cdots, n-1$.

(ii) It is easy to check that $\omega=1-\omega_{1} \omega_{2}>0$ if (Hypothesis 3 ) holds.

Lemma 3. Suppose that condition Hypothesis 3 holds. Let $x, y \in C[0,1]$, then the following systems

$$
\left\{\begin{array}{l}
D_{0^{+}}^{\alpha} u(t)+x(t)=0, \quad t \in(0,1) \\
D_{0^{+}}^{\alpha} v(t)+y(t)=0, \quad t \in(0,1) \\
u^{(j)}(0)=0, j=0,1 \\
v^{(j)}(0)=0, j=0,1 \\
u^{\prime}(1)=\int_{0}^{1} v(t) d \phi(t), v^{\prime}(1)=\int_{0}^{1} u(t) d \psi(t)
\end{array}\right.
$$

have an integral representation

$$
\left\{\begin{array}{l}
u(t)=\int_{0}^{1} G_{1}(t, s) x(s) d s+\int_{0}^{1} I_{1}(t, s) y(s) d s, \\
v(t)=\int_{0}^{1} G_{2}(t, s) y(s) d s+\int_{0}^{1} I_{2}(t, s) x(s) d s,
\end{array}\right.
$$


where $G_{i}(t, s), I_{i}(t, s), i=1,2$ and $G(t, s)$ are the Green's functions associated with the systems (2) given by

$$
\begin{array}{r}
G_{1}(t, s)=\frac{\omega_{1} t^{\alpha-1}}{\omega(\alpha-1)} \int_{0}^{1} G(\tau, s) d \psi(\tau)+G(t, s), \\
G_{2}(t, s)=\frac{\omega_{2} t^{\alpha-1}}{\omega(\alpha-1)} \int_{0}^{1} G(\tau, s) d \phi(\tau)+G(t, s), \\
I_{1}(t, s)=\frac{t^{\alpha-1}}{\omega(\alpha-1)} \int_{0}^{1} G(\tau, s) d \phi(\tau), \\
I_{2}(t, s)=\frac{t^{\alpha-1}}{\omega(\alpha-1)} \int_{0}^{1} G(\tau, s) d \psi(\tau) . \\
G(t, s)= \begin{cases}\frac{t^{\alpha-1}(1-s)^{\alpha-2}-(t-s)^{\alpha-1}}{\Gamma(\alpha)}, & s \leq t, \\
\frac{t^{\alpha-1}(1-s)^{\alpha-2}}{\Gamma(\alpha)}, & t \leq s .\end{cases}
\end{array}
$$

Proof. Based on Lemma 2, we now can reduce (2) to the following equivalent equations

$$
\begin{aligned}
u(t) & =-I_{0^{+}}^{\alpha} x(t)+c_{1} t^{\alpha-1}+c_{2} t^{\alpha-2}+c_{3} t^{\alpha-3} \\
& =-\int_{0}^{t} \frac{(t-s)^{\alpha-1}}{\Gamma(\alpha)} x(s) d s+c_{1} t^{\alpha-1}+c_{2} t^{\alpha-2}+c_{3} t^{\alpha-3}, \\
v(t) & =-I_{0^{+}}^{\alpha} y(t)+c_{1}^{\prime} t^{\alpha-1}+c_{2}^{\prime} t^{\alpha-2}+c_{3}^{\prime} t^{\alpha-3} \\
& =-\int_{0}^{t} \frac{(t-s)^{\alpha-1}}{\Gamma(\alpha)} y(s) d s+c_{1}^{\prime} t^{\alpha-1}+c_{2}^{\prime} t^{\alpha-2}+c_{3}^{\prime} t^{\alpha-3},
\end{aligned}
$$

where $c_{i}, c_{i}^{\prime}, i=1,2,3$ are constants. Next, using the boundary conditions $u^{(j)}(0)=$ $v^{(j)}(0)=0, j=0,1$, we derive $c_{i}=c_{i}^{\prime}=0, i=2,3$, which indicates

$$
\begin{aligned}
& u^{\prime}(t)=-\int_{0}^{t} \frac{(t-s)^{\alpha-2}}{\Gamma(\alpha-1)} x(s) d s+c_{1}(\alpha-1) t^{\alpha-2}, \\
& v^{\prime}(t)=-\int_{0}^{t} \frac{(t-s)^{\alpha-2}}{\Gamma(\alpha-1)} y(s) d s+c_{1}^{\prime}(\alpha-1) t^{\alpha-2} .
\end{aligned}
$$

From the boundary conditions $u^{\prime}(1)=\int_{0}^{1} v(t) d \phi(t), v^{\prime}(1)=\int_{0}^{1} u(t) d \psi(t)$, we immediately obtain

$$
\begin{aligned}
& c_{1}=\int_{0}^{1} \frac{(1-s)^{\alpha-2}}{\Gamma(\alpha)} x(s) d s+\frac{1}{\alpha-1} \int_{0}^{1} v(t) d \phi(t), \\
& c_{1}^{\prime}=\int_{0}^{1} \frac{(1-s)^{\alpha-2}}{\Gamma(\alpha)} y(s) d s+\frac{1}{\alpha-1} \int_{0}^{1} u(t) d \psi(t) .
\end{aligned}
$$

Therefore, we derive 


$$
\begin{aligned}
u(t) & =-\int_{0}^{t} \frac{(t-s)^{\alpha-1}}{\Gamma(\alpha)} x(s) d s+\int_{0}^{1} \frac{t^{\alpha-1}(1-s)^{\alpha-2}}{\Gamma(\alpha)} x(s) d s+\frac{t^{\alpha-1}}{\alpha-1} \int_{0}^{1} v(t) d \phi(t) \\
& =\int_{0}^{1} G(t, s) x(s) d s+\frac{t^{\alpha-1}}{\alpha-1} \int_{0}^{1} v(t) d \phi(t) \\
& =\int_{0}^{1} G(t, s) x(s) d s+\frac{t^{\alpha-1}}{\alpha-1} u^{\prime}(1), \\
v(t)= & -\int_{0}^{t} \frac{(t-s)^{\alpha-1}}{\Gamma(\alpha)} y(s) d s+\int_{0}^{1} \frac{t^{\alpha-1}(1-s)^{\alpha-2}}{\Gamma(\alpha)} y(s) d s+\frac{t^{\alpha-1}}{\alpha-1} \int_{0}^{1} u(t) d \psi(t) \\
& =\int_{0}^{1} G(t, s) y(s) d s+\frac{t^{\alpha-1}}{\alpha-1} \int_{0}^{1} u(t) d \psi(t) \\
& =\int_{0}^{1} G(t, s) y(s) d s+\frac{t^{\alpha-1}}{\alpha-1} v^{\prime}(1) .
\end{aligned}
$$

Integrating (9) and (10) with respect to $d \psi(t)$ and $d \varphi(t)$, respectively, on $[0,1]$ gives

$$
\begin{aligned}
& \int_{0}^{1} u(t) d \psi(t)=u^{\prime}(1) \int_{0}^{1} \frac{t^{\alpha-1}}{\alpha-1} d \psi(t)+\int_{0}^{1} \int_{0}^{1} G(t, s) x(s) d s d \psi(t), \\
& \int_{0}^{1} v(t) d \phi(t)=v^{\prime}(1) \int_{0}^{1} \frac{t^{\alpha-1}}{\alpha-1} d \phi(t)+\int_{0}^{1} \int_{0}^{1} G(t, s) y(s) d s d \phi(t) .
\end{aligned}
$$

Thus, we have

$$
\begin{aligned}
& u^{\prime}(1)=\frac{\omega_{1}}{\omega} \int_{0}^{1} \int_{0}^{1} G(t, s) x(s) d s d \psi(t)+\frac{1}{\omega} \int_{0}^{1} \int_{0}^{1} G(t, s) y(s) d s d \phi(t), \\
& v^{\prime}(1)=\frac{1}{\omega} \int_{0}^{1} \int_{0}^{1} G(t, s) x(s) d s d \psi(t)+\frac{\omega_{2}}{\omega} \int_{0}^{1} \int_{0}^{1} G(t, s) y(s) d s d \phi(t) .
\end{aligned}
$$

Substituting (11) and (12) into (9) and (10), respectively, we can obtain

$$
\begin{aligned}
u(t)= & \frac{\omega_{1} t^{\alpha-1}}{\omega(\alpha-1)} \int_{0}^{1} \int_{0}^{1} G(t, s) x(s) d s d \psi(t) \\
& +\frac{t^{\alpha-1}}{\omega(\alpha-1)} \int_{0}^{1} \int_{0}^{1} G(t, s) y(s) d s d \phi(t)+\int_{0}^{1} G(t, s) x(s) d s, \\
v(t)= & \frac{t^{\alpha-1}}{\omega(\alpha-1)} \int_{0}^{1} \int_{0}^{1} G(t, s) x(s) d s d \psi(t) \\
& +\frac{\omega_{2} t^{\alpha-1}}{\omega(\alpha-1)} \int_{0}^{1} \int_{0}^{1} G(t, s) y(s) d s d \phi(t)+\int_{0}^{1} G(t, s) y(s) d s .
\end{aligned}
$$

Consequently, the solutions of System (2) can be expressed as the following integral forms:

$$
\left\{\begin{array}{l}
u(t)=\int_{0}^{1} G_{1}(t, s) x(s) d s+\int_{0}^{1} I_{1}(t, s) y(s) d s, \\
v(t)=\int_{0}^{1} G_{2}(t, s) y(s) d s+\int_{0}^{1} I_{2}(t, s) x(s) d s .
\end{array}\right.
$$

This completes the proof. 
Remark 2. The function $G(t, s)$ defined by (8) has the following properties (see [32]):

(i) $G(t, s)>0, t, s \in(0,1)$,

(ii) $t^{\alpha-1} G(1, s) \leq G(t, s) \leq G(1, s), t, s \in[0,1]$.

From Remark 2 together with (4)-(8) and condition (Hypothesis 3), it is not difficult to derive

Lemma 4. The functions $G_{1}(t, s), G_{2}(t, s)$ and $I_{1}(t, s), I_{2}(t, s)$ defined by (4), (5)-(7), respectively, have the following properties:

(i) $\varrho t^{\alpha-1}(1-s)^{\alpha-2} s \leq G_{i}(t, s) \leq \rho(1-s)^{\alpha-2} s, i=1,2$,

(ii) $\varrho t^{\alpha-1}(1-s)^{\alpha-2} s \leq I_{i}(t, s) \leq \rho(1-s)^{\alpha-2} s, i=1,2$.

where
$\rho=\max \left\{\frac{\omega_{1}}{\omega} \int_{0}^{1} \frac{1}{\Gamma(\alpha)} d \psi(\tau)+\frac{1}{\Gamma(\alpha)}, \frac{\omega_{2}}{\omega} \int_{0}^{1} \frac{1}{\Gamma(\alpha)} d \phi(\tau)+\frac{1}{\Gamma(\alpha)}, \frac{1}{\omega} \int_{0}^{1} \frac{1}{\Gamma(\alpha)} d \phi(\tau), \frac{1}{\omega} \int_{0}^{1} \frac{1}{\Gamma(\alpha)} d \psi(\tau)\right\}$,
$\varrho=\min \left\{\frac{\omega_{1}}{\omega(\alpha-1)} \int_{0}^{1} \frac{\tau^{\alpha-1}}{\Gamma(\alpha)} d \psi(\tau), \frac{\omega_{2}}{\omega(\alpha-1)} \int_{0}^{1} \frac{\tau^{\alpha-1}}{\Gamma(\alpha)} d \phi(\tau), \frac{1}{\omega(\alpha-1)} \int_{0}^{1} \frac{\tau^{\alpha-1}}{\Gamma(\alpha)} d \phi(\tau), \frac{1}{\omega(\alpha-1)} \int_{0}^{1} \frac{\tau^{\alpha-1}}{\Gamma(\alpha)} d \psi(\tau)\right\}$.

Denote

$$
P=\left\{(u, v) \in E \times E: u(t) \geq \gamma t^{\alpha-1}\|(u, v)\|_{1}, v(t) \geq \gamma t^{\alpha-1}\|(u, v)\|_{1}, t \in[0,1]\right\},
$$

where $\gamma=\frac{\varrho}{\rho} \in(0,1)$. It is easy to verify that $P$ is a positive cone in $E \times E$. Let

$$
P_{r}=\left\{(u, v) \in P:\|(u, v)\|_{1}<r\right\}(r>0)
$$

Define an integral operator $T: P \backslash\{\theta\} \rightarrow P$ by

$$
T(u, v)=\left(T_{1}(u, v), T_{2}(u, v)\right)
$$

where the operators $T_{1}, T_{2}: P \backslash\{\theta\} \rightarrow Q=:\{u \in E: u(t) \geq 0, t \in[0,1]\}$ are defined by

$$
\begin{aligned}
& T_{1}(u, v)(t)=\int_{0}^{1} G_{1}(t, s) f(s, u(s), v(s)) d s+\int_{0}^{1} I_{1}(t, s) g(s, u(s), v(s)) d s, t \in[0,1], \\
& T_{2}(u, v)(t)=\int_{0}^{1} G_{2}(t, s) g(s, u(s), v(s)) d s+\int_{0}^{1} I_{2}(t, s) f(s, u(s), v(s)) d s, t \in[0,1] .
\end{aligned}
$$

Clearly, if $(u, v) \in P \backslash\{\theta\}$ is a fixed point of operator $T$, then $(u, v)$ is a solution of System (1).

Lemma 5. Suppose that (Hypothesis 1)-(Hypothesis 3) hold. Then, the integral operator $T$ : $P \backslash\{\theta\} \rightarrow P$ is well defined.

Proof. We first show that $T(P \backslash\{\theta\}) \subset P$. From that proposed in Lemma 4, for any $t, s, \tau \in[0,1]$, it follows that

$$
\begin{gathered}
G_{i}(t, s) \geq \gamma t^{\alpha-1} G_{i}(\tau, s), I_{i}(t, s) \geq \gamma t^{\alpha-1} I_{i}(\tau, s), i=1,2, \\
G_{1}(t, s) \geq \gamma t^{\alpha-1} I_{2}(\tau, s), I_{1}(t, s) \geq \gamma t^{\alpha-1} G_{2}(\tau, s)
\end{gathered}
$$

and

$$
G_{2}(t, s) \geq \gamma t^{\alpha-1} I_{1}(\tau, s), I_{2}(t, s) \geq \gamma t^{\alpha-1} G_{1}(\tau, s) .
$$


Then, for any $(u, v) \in P \backslash\{\theta\}, t \in[0,1]$, one has

$$
\begin{aligned}
T_{1}(u, v)(t) & =\int_{0}^{1} G_{1}(t, s) f(s, u(s), v(s)) d s+\int_{0}^{1} I_{1}(t, s) g(s, u(s), v(s)) d s \\
& \geq \gamma t^{\alpha-1} \int_{0}^{1} G_{1}(\tau, s) f(s, u(s), v(s)) d s+\gamma t^{\alpha-1} \int_{0}^{1} I_{1}(\tau, s) g(s, u(s), v(s)) d s \\
& =\gamma t^{\alpha-1} T_{1}(u, v)(\tau),
\end{aligned}
$$

and

$$
\begin{aligned}
T_{1}(u, v)(t) & =\int_{0}^{1} G_{1}(t, s) f(s, u(s), v(s)) d s+\int_{0}^{1} I_{1}(t, s) g(s, u(s), v(s)) d s \\
& \geq \gamma t^{\alpha-1} \int_{0}^{1} I_{2}(\tau, s) f(s, u(s), v(s)) d s+\gamma t^{\alpha-1} \int_{0}^{1} G_{2}(\tau, s) g(s, u(s), v(s)) d s \\
& =\gamma t^{\alpha-1} T_{2}(u, v)(\tau),
\end{aligned}
$$

which implies that

$$
T_{1}(u, v)(t) \geq \gamma t^{\alpha-1}\left\|T_{1}(u, v)\right\|, T_{1}(u, v)(t) \geq \gamma t^{\alpha-1}\left\|T_{2}(u, v)\right\| .
$$

Thus, it follows

$$
T_{1}(u, v)(t) \geq \gamma t^{\alpha-1}\left\|\left(T_{1}(u, v), T_{2}(u, v)\right)\right\|_{1} .
$$

Similarly, we can prove

$$
T_{2}(u, v)(t) \geq \gamma t^{\alpha-1}\left\|\left(T_{1}(u, v), T_{2}(u, v)\right)\right\|_{1} .
$$

Consequently, $T(P \backslash\{\theta\}) \subset P$.

For any $(u, v) \in P \backslash\{\theta\}$, one can obtain

$$
\gamma t^{\alpha-1}\|(u, v)\|_{1} \leq u(t), v(t) \leq\|(u, v)\|_{1}, t \in[0,1] .
$$

Choose an appropriate constant $c>0$ such that $c\|(u, v)\|_{1} \leq N, \frac{1}{c}>M$. Thus, by Hypothesis 1 one can have

$$
\begin{aligned}
f(t, u(t), v(t)) & =f\left(t, \frac{1}{c} c u(t), \frac{1}{c} c v(t)\right) \\
& \leq c^{\lambda_{1}}|u(t)|^{\lambda_{1}} c^{\lambda_{2}}|v(t)|^{\lambda_{2}} f\left(t, \frac{1}{c}, \frac{1}{c}\right) \\
& \leq c^{\lambda_{1}-\mu_{1}}|u(t)|^{\lambda_{1}} c^{\lambda_{2}-\mu_{2}}|v(t)|^{\lambda_{2}} f(t, 1,1) \\
& \leq c^{\left(\lambda_{1}+\lambda_{2}\right)-\left(\mu_{1}+\mu_{2}\right)}\|(u, v)\|_{1}^{\lambda_{1}+\lambda_{2}} f(t, 1,1)
\end{aligned}
$$


and

$$
\begin{aligned}
g(t, u(t), v(t)) & =g\left(t, \frac{1}{c} c u(t), \frac{1}{c} c v(t)\right) \\
& \leq c^{\lambda_{3}}|u(t)|^{\lambda_{3}} c^{\lambda_{4}}|v(t)|^{\lambda_{4}} g\left(t, \frac{1}{c}, \frac{1}{c}\right) \\
& \leq c^{\lambda_{3}-\mu_{3}}|u(t)|^{\lambda_{3}} c^{\lambda_{4}-\mu_{4}}|v(t)|^{\lambda_{4}} g(t, 1,1) \\
& \leq c^{\left(\lambda_{3}+\lambda_{4}\right)-\left(\mu_{3}+\mu_{4}\right)}\|(u, v)\|_{1}^{\lambda_{3}+\lambda_{4}} g(t, 1,1) .
\end{aligned}
$$

Hence, for any $t \in[0,1]$, from Hypothesis 2, Hypothesis 3 and (13), (14), it follows that

$$
\begin{aligned}
& T_{i}(u, v)(t) \\
\leq & \rho \int_{0}^{1} s(1-s)^{\alpha-2} f(s, u(s), v(s)) d s+\rho \int_{0}^{1} s(1-s)^{\alpha-2} g(s, u(s), v(s)) d s \\
\leq & \rho c^{\left(\lambda_{1}+\lambda_{2}\right)-\left(\mu_{1}+\mu_{2}\right)}\|(u, v)\|_{1}^{\lambda_{1}+\lambda_{2}} \int_{0}^{1} s(1-s)^{\alpha-2} f(s, 1,1) d s \\
& +\rho c^{\left(\lambda_{3}+\lambda_{4}\right)-\left(\mu_{3}+\mu_{4}\right)}\|(u, v)\|_{1}^{\lambda_{3}+\lambda_{4}} \int_{0}^{1} s(1-s)^{\alpha-2} g(s, 1,1) d s<+\infty, i=1,2 .
\end{aligned}
$$

Consequently, the integral operator $T$ is well defined on $P \backslash\{\theta\}$.

Lemma 6. Suppose that Hypotheses 1-3 hold. Then, for any $0<a<b<+\infty$, the integral operator $T:\left(\overline{P_{b}} \backslash P_{a}\right) \rightarrow P$ is completely continuous.

Proof. It is easy to check that $T\left(\overline{P_{b}} \backslash P_{a}\right) \subset P$ according to Lemma 5 . Now, we show that the operator $T$ is compact. For any bounded subset $B \subset \overline{P_{b}} \backslash P_{a}$, we can choose a suitable real constant $c \in(0,1)$ such that

$$
\|(u, v)\|_{1} \leq \frac{N}{c}, \frac{1}{c}>M,
$$

for all $(u, v) \in B$. By virtue of the proof processes in Lemma 5, one can obtain

$$
\begin{aligned}
T_{i}(u, v)(t) & \leq \rho \int_{0}^{1} s(1-s)^{\alpha-2} f(s, u(s), v(s)) d s+\rho \int_{0}^{1} s(1-s)^{\alpha-2} g(s, u(s), v(s)) d s \\
& \leq \rho c^{\left(\lambda_{1}+\lambda_{2}\right)-\left(\mu_{1}+\mu_{2}\right)} a^{\lambda_{1}+\lambda_{2}} \int_{0}^{1} s(1-s)^{\alpha-2} f(s, 1,1) d s \\
& +\rho c^{\left(\lambda_{3}+\lambda_{4}\right)-\left(\mu_{3}+\mu_{4}\right)} a^{\lambda_{3}+\lambda_{4}} \int_{0}^{1} s(1-s)^{\alpha-2} g(s, 1,1) d s<+\infty, i=1,2
\end{aligned}
$$

which illustrates that $T(B)$ is uniformly bounded. 
Next, we are ready to show that $T(B)$ is equicontinuous on $[0,1]$. From the proof of Lemma 3, for any $(x, y) \in B, t \in[0,1]$, it follows that

$$
\begin{aligned}
T_{1}(u, v)(t)= & \int_{0}^{1} G_{1}(t, s) f(s, u(s), v(s)) d s+\int_{0}^{1} I_{1}(t, s) g(s, u(s), v(s)) d s \\
= & \frac{\omega_{1} t^{\alpha-1}}{\omega(\alpha-1)} \int_{0}^{1}\left(\int_{0}^{1} G(\tau, s) d \psi(\tau)\right) f(s, u(s), v(s)) d s \\
& +\int_{0}^{t} \frac{t^{\alpha-1}(1-s)^{\alpha-2}-(t-s)^{\alpha-1}}{\Gamma(\alpha)} f(s, u(s), v(s)) d s \\
& +\int_{t}^{1} \frac{t^{\alpha-1}(1-s)^{\alpha-2}}{\Gamma(\alpha)} f(s, u(s), v(s)) d s \\
& +\frac{t^{\alpha-1}}{\omega(\alpha-1)} \int_{0}^{1}\left(\int_{0}^{1} G(\tau, s) d \phi(\tau)\right) g(s, u(s), v(s)) d s .
\end{aligned}
$$

Differentiating with respect to $t$ and combining this with Hypothesis 1 , we have

$$
\begin{aligned}
& \left|T_{1}(u, v)^{\prime}(t)\right|=\frac{\omega_{1} t^{\alpha-2}}{\omega} \int_{0}^{1}\left(\int_{0}^{1} G(\tau, s) d \psi(\tau)\right) f(s, u(s), v(s)) d s \\
& +\int_{0}^{t} \frac{(\alpha-1) t^{\alpha-2}(1-s)^{\alpha-2}-(\alpha-1)(t-s)^{\alpha-2}}{\Gamma(\alpha)} f(s, u(s), v(s)) d s \\
& +\int_{t}^{1} \frac{(\alpha-1) t^{\alpha-2}(1-s)^{\alpha-2}}{\Gamma(\alpha)} f(s, u(s), v(s)) d s \\
& +\frac{t^{\alpha-2}}{\omega} \int_{0}^{1}\left(\int_{0}^{1} G(\tau, s) d \phi(\tau)\right) g(s, u(s), v(s)) d s \\
& \leq \frac{\omega_{1}}{\omega \Gamma(\alpha)} \int_{0}^{1} d \psi(\tau) \int_{0}^{1} s(1-s)^{\alpha-2} f(s, u(s), v(s)) d s \\
& +\int_{0}^{t} \frac{(\alpha-1) t^{\alpha-2}(1-s)^{\alpha-2}-(\alpha-1)(t-s)^{\alpha-2}}{\Gamma(\alpha)} f(s, u(s), v(s)) d s \\
& +\int_{t}^{1} \frac{(\alpha-1) t^{\alpha-2}(1-s)^{\alpha-2}}{\Gamma(\alpha)} f(s, u(s), v(s)) d s \\
& +\frac{1}{\omega \Gamma(\alpha)} \int_{0}^{1} d \phi(\tau) \int_{0}^{1} s(1-s)^{\alpha-2} g(s, u(s), v(s)) d s \\
& \leq c^{\left(\lambda_{1}+\lambda_{2}\right)-\left(\mu_{1}+\mu_{2}\right)} a^{\lambda_{1}+\lambda_{2}}\left(\frac{\omega_{1}}{\omega \Gamma(\alpha)} \int_{0}^{1} d \psi(\tau) \int_{0}^{1} s(1-s)^{\alpha-2} f(s, 1,1) d s\right. \\
& +\int_{0}^{t} \frac{(\alpha-1) t^{\alpha-2}(1-s)^{\alpha-2}-(\alpha-1)(t-s)^{\alpha-2}}{\Gamma(\alpha)} f(s, 1,1) d s \\
& \left.+\int_{t}^{1} \frac{(\alpha-1) t^{\alpha-2}(1-s)^{\alpha-2}}{\Gamma(\alpha)} f(s, 1,1) d s\right) \\
& +c^{\left(\lambda_{3}+\lambda_{4}\right)-\left(\mu_{3}+\mu_{4}\right)} a^{\lambda_{3}+\lambda_{4}} \frac{1}{\omega \Gamma(\alpha)} \int_{0}^{1} d \phi(\tau) \int_{0}^{1} s(1-s)^{\alpha-2} g(s, 1,1) d s .
\end{aligned}
$$


Let

$$
\begin{aligned}
\Theta(t)= & c^{\left(\lambda_{1}+\lambda_{2}\right)-\left(\mu_{1}+\mu_{2}\right)} a^{\lambda_{1}+\lambda_{2}}\left(\frac{\omega_{1}}{\omega \Gamma(\alpha)} \int_{0}^{1} d \psi(\tau) \int_{0}^{1} s(1-s)^{\alpha-2} f(s, 1,1) d s\right. \\
& +\int_{0}^{t} \frac{(\alpha-1) t^{\alpha-2}(1-s)^{\alpha-2}-(\alpha-1)(t-s)^{\alpha-2}}{\Gamma(\alpha)} f(s, 1,1) d s \\
& \left.+\int_{t}^{1} \frac{(\alpha-1) t^{\alpha-2}(1-s)^{\alpha-2}}{\Gamma(\alpha)} f(s, 1,1) d s\right) \\
& +c^{\left(\lambda_{3}+\lambda_{4}\right)-\left(\mu_{3}+\mu_{4}\right)} a^{\lambda_{3}+\lambda_{4}} \frac{1}{\omega \Gamma(\alpha)} \int_{0}^{1} d \phi(\tau) \int_{0}^{1} s(1-s)^{\alpha-2} g(s, 1,1) d s .
\end{aligned}
$$

Exchanging the integral order and using condition Hypothesis 2, we derive that

$$
\begin{aligned}
\int_{0}^{1} \Theta(t) d t \leq & c^{\left(\lambda_{1}+\lambda_{2}\right)-\left(\mu_{1}+\mu_{2}\right)} a^{\lambda_{1}+\lambda_{2}}\left(\frac{\omega_{1}}{\omega \Gamma(\alpha)} \int_{0}^{1} d \psi(\tau) \int_{0}^{1} s(1-s)^{\alpha-2} f(s, 1,1) d s\right. \\
& +\int_{0}^{1}\left(\frac{s(1-s)^{\alpha-2}}{\Gamma(\alpha)}-\frac{s^{\alpha-1}(1-s)^{\alpha-2}}{\Gamma(\alpha)}\right) f(s, 1,1) d s \\
& \left.+\int_{0}^{1} \frac{(\alpha-1) s(1-s)^{\alpha-2}}{\Gamma(\alpha)} f(s, 1,1) d s\right) \\
& +c^{\left(\lambda_{3}+\lambda_{4}\right)-\left(\mu_{3}+\mu_{4}\right)} a^{\lambda_{3}+\lambda_{4}} \frac{1}{\omega \Gamma(\alpha)} \int_{0}^{1} d \phi(\tau) \int_{0}^{1} s(1-s)^{\alpha-2} g(s, 1,1) d s<+\infty
\end{aligned}
$$

The absolute continuity of the integral yields that $T_{1}(B)$ is equicontinuous on $[0,1]$. From this together with the boundedness of $T_{1}(B)$ and the Arzelà-Ascoli theorem, it follows that $T_{1}(B)$ is a relatively compact set. Similarly, we can show that $T_{2}(B)$ is also a relatively compact set. Consequently, $T(B)$ is relatively compact.

Finally, we shall show that $T$ is continuous. For this purpose, we let $\left(u_{m}, v_{m}\right),\left(u_{0}, v_{0}\right) \in$ $\overline{P_{b}} \backslash P_{a}$ such that $\left\|\left(u_{m}, v_{m}\right)-\left(u_{0}, v_{0}\right)\right\|_{1} \rightarrow 0$ as $m \rightarrow+\infty$. Choose an appropriate real constant $c \in(0,1)$ such that $c b \leq N, \frac{1}{c} \geq M$. From this together with (Hypothesis 1), it follows that

$$
\begin{aligned}
& f\left(t, u_{m}(t), v_{m}(t)\right) \leq c^{\left(\lambda_{1}+\lambda_{2}\right)-\left(\mu_{1}+\mu_{2}\right)} a^{\lambda_{1}+\lambda_{2}} f(t, 1,1), m=0,1,2, \cdots, \\
& g\left(t, u_{m}(t), v_{m}(t)\right) \leq c^{\left(\lambda_{3}+\lambda_{4}\right)-\left(\mu_{3}+\mu_{4}\right)} a^{\lambda_{3}+\lambda_{4}} g(t, 1,1), m=0,1,2, \cdots,
\end{aligned}
$$

and

$$
\begin{aligned}
& \left|T_{1}\left(u_{m}, v_{m}\right)(t)-T_{1}\left(u_{0}, v_{0}\right)(t)\right| \\
\leq & \int_{0}^{1} G_{1}(t, s)\left|f\left(s, u_{m}(s), v_{m}(s)\right)-f\left(s, u_{0}(s), v_{0}(s)\right)\right| d s \\
& +\int_{0}^{1} I_{1}(t, s)\left|g\left(s, u_{m}(s), v_{m}(s)\right)-g\left(s, u_{0}(s), v_{0}(s)\right)\right| d s \\
\leq & \rho \int_{0}^{1} s(1-s)^{\alpha-2}\left|f\left(s, u_{m}(s), v_{m}(s)\right)-f\left(s, u_{0}(s), v_{0}(s)\right)\right| d s \\
& +\rho \int_{0}^{1} s(1-s)^{\alpha-2}\left|g\left(s, u_{m}(s), v_{m}(s)\right)-g\left(s, u_{0}(s), v_{0}(s)\right)\right| d s .
\end{aligned}
$$


By the Lebesgue dominated convergence theorem, one can have

$$
T_{1}\left(u_{m}, v_{m}\right)(t) \rightarrow T_{1}\left(u_{0}, v_{0}\right)(t), m \rightarrow+\infty .
$$

Hence, the Arzelà-Ascoli theorem guarantees that

$$
\left\|T_{1}\left(u_{m}, v_{m}\right)-T_{1}\left(u_{0}, v_{0}\right)\right\| \rightarrow 0, m \rightarrow+\infty .
$$

In the same way, we can prove that

$$
\left\|T_{2}\left(u_{m}, v_{m}\right)-T_{2}\left(u_{0}, v_{0}\right)\right\| \rightarrow 0, m \rightarrow+\infty .
$$

Then, we have

$$
\left\|T\left(u_{m}, v_{m}\right)-T\left(u_{0}, v_{0}\right)\right\|_{1} \rightarrow 0, m \rightarrow+\infty,
$$

which implies that $T:\left(\overline{P_{b}} \backslash P_{a}\right) \rightarrow P$ is continuous. Consequently, $T:\left(\overline{P_{b}} \backslash P_{a}\right) \rightarrow P$ is completely continuous. The result of Lemma 6 follows.

Remark 3. From the proof of Lemma 5 and 6, we can see that singularity difficulty in proving operator $T$ to be completely continuous is overcome by the special construction of cone $P$ and the integral condition (Hypothesis 2) imposed on the nonlinear terms.

Lemma 7 (Guo-Krasnoselskii's fixed point theorem (see [31])). Let $E$ be a Banach space, $P \subseteq E$ a cone, and $\Omega_{1}, \Omega_{2}$ two bounded open balls of E centered at the origin with $\bar{\Omega}_{1} \subset \Omega_{2}$. Suppose that $T: P \cap\left(\bar{\Omega}_{2} \backslash \Omega_{1}\right) \rightarrow P$ is a completely continuous operator such that either (i) $\|T x\| \leq\|x\|, x \in P \cap \partial \Omega_{1}$ and $\|T x\| \geq\|x\|, x \in P \cap \partial \Omega_{2}$, or (ii) $\|T x\| \geq\|x\|, x \in P \cap \partial \Omega_{1}$ and $\|T x\| \leq\|x\|, x \in P \cap \partial \Omega_{2}$, holds. Then $T$ has a fixed point in $P \cap\left(\bar{\Omega}_{2} \backslash \Omega_{1}\right)$.

\section{Main Results}

Theorem 1. Suppose that Hypotheses 1-3 hold. Then, if $0<\mu_{0}<1$, the system (1) has at least one positive solution.

Proof. First, we claim that there exists a sufficiently small constant $r>0$ such that

$$
\|T(u, v)\|_{1} \geq\|(u, v)\|_{1}, \forall(u, v) \in \partial P_{r} .
$$

Let $c=\frac{1}{M}$, and choose the constant $r>0$ such that

$$
r=\min \left\{M N, \frac{1}{2},\left(\frac{\varrho \gamma}{4^{\alpha-1}} c^{1-\lambda_{0}}\left(\int_{0}^{1} s^{\alpha}(1-s)^{\alpha-2} f(s, 1,1) d s+\int_{0}^{1} s^{\alpha}(1-s)^{\alpha-2} g(s, 1,1) d s\right)\right)^{\frac{1}{1-\mu_{0}}}\right\},
$$

which yields that

$$
c u(s), c v(s) \leq c\|(u, v)\|_{1}=c r \leq N, \forall(u, v) \in \partial P_{r}, s \in[0,1],
$$

and

$$
\gamma r s^{\alpha-1} \leq u(s), v(s) \leq r, \forall(u, v) \in \partial P_{r}, s \in[0,1] .
$$

From Hypothesis 1, Hypothesis 2, and Lemma 4, we can derive that 


$$
\begin{aligned}
T_{i}(u, v)(t) \geq & \frac{\varrho}{4^{\alpha-1}}\left(\int_{0}^{1} s(1-s)^{\alpha-2} f\left(s, \frac{1}{c} c u(s), \frac{1}{c} c v(s)\right) d s\right. \\
& \left.+\int_{0}^{1} s(1-s)^{\alpha-2} g\left(s, \frac{1}{c} c u(s), \frac{1}{c} c v(s)\right) d s\right) \\
\geq & \frac{\varrho}{4^{\alpha-1}}\left(r^{\mu_{1}+\mu_{2}} c^{\left(\mu_{1}+\mu_{2}\right)-\left(\lambda_{1}+\lambda_{2}\right)} \gamma^{\mu_{1}+\mu_{2}} \int_{0}^{1} s^{1+(\alpha-1)\left(\mu_{1}+\mu_{2}\right)} f(s, 1,1) d s\right. \\
& \left.+r^{\mu_{3}+\mu_{4}} c^{\left(\mu_{3}+\mu_{4}\right)-\left(\lambda_{3}+\lambda_{4}\right)} \gamma^{\mu_{3}+\mu_{4}} \int_{0}^{1} s^{1+(\alpha-1)\left(\mu_{3}+\mu_{4}\right)} g(s, 1,1) d s\right) \\
\geq & \frac{\varrho \gamma}{4^{\alpha-1}} c^{1-\lambda_{0}} r^{\mu_{0}}\left(\int_{0}^{1} s^{\alpha}(1-s)^{\alpha-2} f(s, 1,1) d s+\int_{0}^{1} s^{\alpha}(1-s)^{\alpha-2} g(s, 1,1) d s\right) \\
\geq & r=\|(u, v)\|_{1}, i=1,2, t \in\left[\frac{1}{4}, \frac{3}{4}\right], \forall(u, v) \in \partial P_{r},
\end{aligned}
$$

which implies that

$$
\|T(u, v)\|_{1} \geq\|(u, v)\|_{1}, \forall(u, v) \in \partial P_{r} .
$$

Next, we shall show that there exists a sufficiently large constant $R>r$ such that

$$
\|T(u, v)\|_{1} \leq\|(u, v)\|_{1}, \forall(u, v) \in \partial P_{R} .
$$

To this end, we can choose

$$
\begin{aligned}
R=\max \left\{\left(\rho\left(\frac{N}{2}\right)^{\lambda_{0}-\mu_{0}}\left(\int_{0}^{1} s(1-s)^{\alpha-2} f(s, 1,1) d s+\int_{0}^{1} s(1-s)^{\alpha-2} g(s, 1,1) d s\right)\right)^{\frac{1}{1-\mu_{0}}}, 1, \frac{M N}{2}\right\} . \\
\text { Let } c=\frac{N}{2 R} \text {. Then, } \frac{1}{c}=\frac{2 R}{N} \geq M, \text { and } \\
\quad c u(s), c v(s) \leq c\|(u, v)\|_{1}=c R=\frac{N}{2}<N, \forall(u, v) \in \partial P_{R}, s \in[0,1],
\end{aligned}
$$

From Hypothesis 1, Hypothesis 2, and Lemma 4, it follows that 


$$
\begin{aligned}
T_{i}(u, v)(t) \leq & \int_{0}^{1} \rho s(1-s)^{\alpha-2} f\left(s, \frac{1}{c} c u(s), \frac{1}{c} c v(s)\right) d s \\
& +\int_{0}^{1} \rho s(1-s)^{\alpha-2} g\left(s, \frac{1}{c} c u(s), \frac{1}{c} c v(s)\right) d s \\
\leq & \rho R^{\lambda_{1}+\lambda_{2}} c^{\left(\lambda_{1}+\lambda_{2}\right)-\left(\mu_{1}+\mu_{2}\right)} \int_{0}^{1} s(1-s)^{\alpha-2} f(s, 1,1) d s \\
& +\rho R^{\lambda_{3}+\lambda_{4}} c^{\left(\lambda_{3}+\lambda_{4}\right)-\left(\mu_{3}+\mu_{4}\right)} \int_{0}^{1} s(1-s)^{\alpha-2} g(s, 1,1) d s \\
= & \rho R^{\mu_{1}+\mu_{2}}\left(\frac{N}{2}\right)^{\left(\lambda_{1}+\lambda_{2}\right)-\left(\mu_{1}+\mu_{2}\right)} \int_{0}^{1} s(1-s)^{\alpha-2} f(s, 1,1) d s \\
& +\rho R^{\mu_{3}+\mu_{4}}\left(\frac{N}{2}\right)^{\left(\lambda_{3}+\lambda_{4}\right)-\left(\mu_{3}+\mu_{4}\right)} \int_{0}^{1} s(1-s)^{\alpha-2} g(s, 1,1) d s \\
\leq & \rho R^{\mu_{0}}\left(\frac{N}{2}\right)^{\lambda_{0}-\mu_{0}}\left(\int_{0}^{1} s(1-s)^{\alpha-2} f(s, 1,1) d s+\int_{0}^{1} s(1-s)^{\alpha-2} g(s, 1,1) d s\right) \\
\leq & R=\|(u, v)\|_{1}, i=1,2, t \in[0,1], \forall(u, v) \in \partial P_{R},
\end{aligned}
$$

and then this indicates that

$$
\|T(u, v)\|_{1} \leq\|(u, v)\|_{1}, \forall(u, v) \in \partial P_{R}
$$

Therefore, Combining (15), (16) together with Lemma 7 yields our result. This completes the proof.

Theorem 2. Suppose that Hypotheses $1-3$ hold. In addition, there exists a real constant $\sigma \in(0,1)$ such that the following two conditions are satisfied:

Hypothesis 4. $\Lambda:=\max \left\{1, \frac{M N}{\gamma \sigma^{\alpha-1}}\right\}<\Delta^{\frac{1}{1-\lambda_{0}}}$, where

$$
\Delta=\varrho \sigma^{\left(1+\lambda^{0}\right)(\alpha-1)} N^{\mu_{0}-\lambda_{0}} \gamma^{\lambda^{0}} \int_{\sigma}^{1} s(1-s)^{\alpha-2}(f(s, 1,1)+g(s, 1,1)) d s,
$$

Hypothesis 5. $\int_{0}^{1} s^{1+(\alpha-1) \lambda_{0}}(1-s)^{\alpha-2} f(s, 1,1) d s+\int_{0}^{1} s^{1+(\alpha-1) \lambda_{0}}(1-s)^{\alpha-2} g(s, 1,1) d s<$ $\frac{\omega^{\mu_{0}-\lambda_{0}}}{\rho \gamma^{\lambda_{0}}}$.

Then System (1) has at least two positive solutions $\left(u_{i}, v_{i}\right) \in P, i=1,2$ such that

$$
0<\left\|\left(u_{1}, v_{1}\right)\right\|_{1}<1<\left\|\left(u_{2}, v_{2}\right)\right\|_{1}<+\infty
$$

Proof. We first prove that there exists a sufficiently small constant $r>0$ such that

$$
\|T(u, v)\|_{1} \geq\|(u, v)\|_{1}, \forall(u, v) \in \partial P_{r}
$$

As already done in the proof of Theorem 1, this can be derived similarly, and thus the proof process is omitted here. 
Next, we shall show that

$$
\|T(u, v)\|_{1}<\|(u, v)\|_{1}, \forall(u, v) \in \partial P_{1} .
$$

Noticing that $\omega=\min \left\{N, \frac{1}{M}\right\}$, and thus we have

$$
\omega u(s), \omega v(s) \leq N, \frac{1}{\omega} \geq M, \forall(u, v) \in \partial P_{1}, s \in[0,1],
$$

and

$$
\gamma s^{\alpha-1} \leq u(s), v(s) \leq 1, \forall(u, v) \in \partial P_{1}, s \in[0,1] .
$$

From Hypothesis 1, Hypothesis 2, and Lemma 4 together with Hypothesis 5, we can deduce that

$$
\begin{aligned}
T_{i}(u, v)(t) \leq & \int_{0}^{1} \rho s(1-s)^{\alpha-2} f\left(s, \frac{1}{\omega} \mathcal{\omega} u(s), \frac{1}{\omega} \omega v(s)\right) d s \\
& +\int_{0}^{1} \rho s(1-s)^{\alpha-2} g\left(s, \frac{1}{\omega} \omega u(s), \frac{1}{\omega} \omega v(s)\right) d s \\
\leq & \rho \gamma^{\lambda_{1}+\lambda_{2}} \omega^{\left(\lambda_{1}+\lambda_{2}\right)-\left(\mu_{1}+\mu_{2}\right)} \int_{0}^{1} s^{1+(\alpha-1)\left(\lambda_{1}+\lambda_{2}\right)}(1-s)^{\alpha-2} f(s, 1,1) d s \\
& +\rho \gamma^{\lambda_{3}+\lambda_{4}} \mathscr{\omega}^{\left(\lambda_{3}+\lambda_{4}\right)-\left(\mu_{3}+\mu_{4}\right)} \int_{0}^{1} s^{1+(\alpha-1)\left(\lambda_{3}+\lambda_{4}\right)}(1-s)^{\alpha-2} g(s, 1,1) d s \\
\leq & \rho \gamma^{\lambda_{0}} \omega^{\lambda_{0}-\mu_{0}} \int_{0}^{1} s^{1+(\alpha-1) \lambda_{0}}(1-s)^{\alpha-2} f(s, 1,1) d s \\
& +\rho \gamma^{\lambda_{0}} \mathcal{\omega}^{\lambda_{0}-\mu_{0}} \int_{0}^{1} s^{1+(\alpha-1) \lambda_{0}}(1-s)^{\alpha-2} g(s, 1,1) d s \\
< & 1=\|(u, v)\|_{1}, i=1,2, t \in[0,1], \forall(u, v) \in \partial P_{1},
\end{aligned}
$$

which illustrates that

$$
\|T(u, v)\|_{1}<\|(u, v)\|_{1}, \forall(u, v) \in \partial P_{1} .
$$

Notably, System (1) has no positive solution on $\partial P_{1}$.

Finally, we prove that there exists a sufficiently large constant $R>1$ such that

$$
\|T(u, v)\|_{1} \geq\|(u, v)\|_{1}, \forall(u, v) \in \partial P_{R} .
$$

Let $c=\frac{1}{N}$ and choose $R>0$ such that

$$
R=\Lambda+\theta\left(\Delta^{\frac{1}{1-\lambda_{0}}}-\Lambda\right), 0<\theta<1
$$

Then, it follows that

$$
c u(s), c v(s) \geq c \gamma s^{\alpha-1}\|(u, v)\|_{1} \geq c \gamma \sigma^{\alpha-1} R>M, \forall(u, v) \in \partial P_{R}, s \in[\sigma, 1] .
$$


From this and Lemma 4 together with Hypothesis 1, Hypothesis 2, and Hypothesis 4, one can have

$$
\begin{aligned}
T_{i}(u, v)(t) \geq & \varrho \sigma^{\alpha-1}\left(\int_{\sigma}^{1} s(1-s)^{\alpha-2} f\left(s, \frac{1}{c} c u(s), \frac{1}{c} c v(s)\right) d s\right. \\
& \left.+\int_{\sigma}^{1} s(1-s)^{\alpha-2} g\left(s, \frac{1}{c} c u(s), \frac{1}{c} c v(s)\right) d s\right) \\
\geq & \varrho \sigma^{\alpha-1}\left(c^{\left(\lambda_{1}+\lambda_{2}\right)-\left(\mu_{1}+\mu_{2}\right)}\left(\gamma \sigma^{\alpha-1} R\right)^{\lambda_{1}+\lambda_{2}} \int_{\sigma}^{1} s(1-s)^{\alpha-2} f(s, 1,1) d s\right. \\
& \left.+c^{\left(\lambda_{3}+\lambda_{4}\right)-\left(\mu_{3}+\mu_{4}\right)}\left(\gamma \sigma^{\alpha-1} R\right)^{\lambda_{3}+\lambda_{4}} \int_{\sigma}^{1} s(1-s)^{\alpha-2} g(s, 1,1) d s\right) \\
\geq & \varrho \sigma^{\left(1+\lambda^{0}\right)(\alpha-1)} N^{\mu_{0}-\lambda_{0}} \gamma^{\lambda^{0}} \int_{\sigma}^{1} s(1-s)^{\alpha-2}(f(s, 1,1)+g(s, 1,1)) d s \cdot R^{\lambda_{0}} \\
\geq & R=\|(u, v)\|_{1}, i=1,2, t \in[\sigma, 1], \forall(u, v) \in \partial P_{R},
\end{aligned}
$$

which indicates that

$$
\|T(u, v)\|_{1} \geq\|(u, v)\|_{1}, \forall(u, v) \in \partial P_{R} .
$$

Thus, in view of (17) and (18), by Lemma 7, System (1) has at least two positive solutions $\left(u_{i}, v_{i}\right) \in P, i=1,2$ such that

$$
0<\left\|\left(u_{1}, v_{1}\right)\right\|_{1}<1<\left\|\left(u_{2}, v_{2}\right)\right\|_{1}<+\infty .
$$

The conclusion of this theorem follows.

Remark 4. (i) The singularity of the studied nonlinear terms with respect to variables $u$ and $v$ could be large enough; that is, if the nonlinear terms contain $\frac{1}{u^{\lambda}}, \frac{1}{v^{\mu}}$, then $\lambda, \mu$ could be large enough positive numbers. (ii) Although the system studied in [33] is more general, it does not have singularity. Then the conditions imposed on nonlinear functions and the techniques employed in this paper are completely different from it. Authors in [27] only discussed the singularity at $t=0,1$, and the assumptions on the nonlinear functions to overcome the singularity were relatively strong. For details, the condition (Hypothesis 4) in [27] is that

$$
\int_{0}^{1} t(1-t)^{\alpha-1} f(t, u, v) d t<+\infty, \int_{0}^{1} t(1-t)^{\alpha-1} g(t, u, v) d t<+\infty,
$$

for any $u, v$. However, our condition (Hypothesis 2) to deal with the singularity is that

$$
\int_{0}^{1} t(1-t)^{\alpha-2} f(t, 1,1) d t<+\infty, \int_{0}^{1} t(1-t)^{\alpha-2} g(t, 1,1) d t<+\infty,
$$

which is a more weaker condition than that (Hypothesis 4) in [27]. (iii) Compared with [27,33], we get the existence of not only at least one positive solution but also the existence of at least two positive solutions of the considered singular system.

\section{An Example}

In this section, we present an example to illustrate the validity of the proposed result. 
Example 1. Consider the following boundary value problems of fractional singular differential systems with coupled integral boundary value conditions:

$$
\left\{\begin{array}{l}
D_{0^{+}}^{\frac{5}{2}} u(t)+\frac{e^{t}}{t \sqrt{1-t}} u^{-5} v^{-4}+\frac{\sin t}{\sqrt[3]{t^{2}}(1-t)} u^{\frac{1}{3}} v^{\frac{1}{4}}=0, \quad t \in(0,1), \\
D_{0^{+}}^{\frac{5}{2}} v(t)+\frac{e^{t}}{\sqrt{t}} u^{-6} v^{-5}+\frac{\cos t}{\sqrt{t(1-t)}} u^{\frac{1}{3}} v^{\frac{1}{4}}=0, t \in(0,1), \\
u^{(j)}(0)=0, j=0,1, \\
v^{(j)}(0)=0, j=0,1, \\
u^{\prime}(1)=\int_{0}^{1} v(t) d \sqrt{t}, v^{\prime}(1)=\int_{0}^{1} u(t) d t^{2} .
\end{array}\right.
$$

We conclude that System (19) has at least one positive solution.

Proof. Notably, (19) can be regarded as a boundary value problem of the form (1), where

$$
\begin{gathered}
f(t, u, v)=\frac{e^{t}}{t \sqrt{1-t}} u^{-5} v^{-4}+\frac{\sin t}{\sqrt[3]{t^{2}}(1-t)} u^{\frac{1}{3}} v^{\frac{1}{4}}, \\
g(t, u, v)=\frac{e^{t}}{\sqrt{t}} u^{-6} v^{-5}+\frac{\cos t}{\sqrt{t(1-t)}} u^{\frac{1}{3}} v^{\frac{1}{4}}, \\
\phi(t)=\sqrt{t}, \psi(t)=t^{2},
\end{gathered}
$$

and

$$
\begin{gathered}
\lambda_{1}=-7, \lambda_{2}=-6, \lambda_{3}=-8, \lambda_{4}=-7, \\
\mu_{1}=\frac{1}{2}, \mu_{2}=\frac{1}{3}, \mu_{3}=\frac{1}{3}, \mu_{4}=\frac{1}{2} .
\end{gathered}
$$

Straightforward calculation gives

$$
\int_{0}^{1} \frac{1}{\sqrt{s(1-s)}} d s=\pi, \int_{0}^{1} \sqrt{s(1-s)} d s=\frac{\pi}{8},
$$

which can derive

$$
\omega_{1}=\frac{1}{6}, \omega_{2}=\frac{8}{21}, \omega=1-\omega_{1} \omega_{2}=\frac{59}{63}
$$

and

$$
\begin{aligned}
& \int_{0}^{1} s(1-s)^{\alpha-2} f(s, 1,1) d s \leq \int_{0}^{1} e^{s} d s+\int_{0}^{1} \frac{1}{\sqrt{s(1-s)}} d s=e-1+\pi<+\infty, \\
& \int_{0}^{1} s(1-s)^{\alpha-2} g(s, 1,1) d s \leq e \int_{0}^{1} \sqrt{s(1-s)} d s+\int_{0}^{1} \sqrt{s} d s=\frac{\pi e}{8}+\frac{2}{3}<+\infty .
\end{aligned}
$$

Hereto, we can infer that $f(t, u, v)$ and $g(t, u, v)$ satisfy conditions Hypothesis 1-Hypothesis 3. Hence, Theorem 1 guarantees that System (19) has at least one positive solution.

\section{Conclusions}

In the present paper, we deal with the existence and multiplicity of positive solutions for a class of nonlinear singular fractional differential systems with Riemann-Stieltjes coupled integral boundary value conditions. More precisely, in order to conquer the difficulties caused by singularity not only at $t=0,1$ but also at $u, v=0$ in the nonlinear terms, a suitable cone is constructed through the good properties of Green's functions associated with the systems, and some necessary assumptions are imposed on the nonlinear functions. Compared with other basic methods to deal with the singularity, such as sequential techniques of approximate system and the mixed monotone method, the idea adopted in this 
paper is relatively concise and has its own advantages, since some superlinear or sublinear conditions and monotonicity conditions are no longer needed for nonlinear terms.

Author Contributions: Conceptualization and Visualization, D.Z.; Formal analysis and Investigation, J.M.;Writing original draft and Investigation, D.Z and J.M. All authors have read and agreed to the published version of the manuscript.

Funding: Research supported by the project of Shandong Province Higher Educational Science and Technology Program of China under the grant J18KA233, the Natural Science Fund for Distinguished Young Scholars of Shandong Province under grant JQ201613.

Institutional Review Board Statement: Not applicable.

Informed Consent Statement: Not applicable.

Data Availability Statement: No new data were created or analyzed in this study.

Conflicts of Interest: The authors declare that there are no conflicts of interest regarding the publication of this paper.

\section{References}

1. Cheng, H.; Yuan, R. The stability of the equilibria of the Allen-Cahn equation with fractional diffusion. Appl. Anal. 2019, 98, 600-610. [CrossRef]

2. Jia, J.; Wang, H. A fast finite difference method for distributed-order space-fractional partial differential equations on convex domains. Comput. Math. Appl. 2018, 75, 2031-2043. [CrossRef]

3. Jia, J.; Wang, H. A fast finite volume method for conservative space-time fractional diffusion equations discretized on space-time locally refined meshes. Comput. Math. Appl. 2019, 78, 1345-1356. [CrossRef]

4. Li, S.; Zhou, Z. Fractional spectral collocation method for optimal control problem governed by space fractional diffusion equation. Appl. Math. Comput. 2019, 350, 331-347. [CrossRef]

5. Mao, J.; Zhao, D. Multiple positive solutions for nonlinear fractional differential equations with integral boundary value conditions and a parameter. J. Funct. Space. 2019, 2019, 2787569. [CrossRef]

6. Qi, T.; Liu, Y.; Cui, Y. Existence of solutions for a class of coupled fractional differential systems with nonlocal boundary conditions. J. Funct. Space. 2017, 2017, 6703860. [CrossRef]

7. Qi, T.; Liu, Y.; Zou, Y. Existence result for a class of coupled fractional differential systems with integral boundary value conditions. J. Nonlinear Sci. Appl. 2017, 10, 4034-4045. [CrossRef]

8. Tian, Y.; Zhao, S. Existence of solutions for perturbed fractional equations with two competing weighted nonlinear terms. Bound. Value. Probl. 2018, 2018, 154. [CrossRef]

9. Tian, Y. Some results on the eigenvalue problem for a fractional elliptic equation. Bound. Value. Probl. 2019, 2019, 13. [CrossRef]

10. Zhao, D.; Liu, Y. Multiple positive solutions for nonlinear fractional boundary value problems. Sci. World J. 2013, 2013, 473828. [CrossRef]

11. Li, H.; Wu, X.; Zhang, J. Numerical solution of the time-fractional sub-diffusion equation on an unbounded domain in twodimensional space. Eur. Asian J. Appl. Math. 2017, 7, 439-454. [CrossRef]

12. Liu, Y. Bifurcation techniques for a class of boundary value problems of fractional impulsive differential equations. J. Nonlinear Sci. Appl. 2015, 8, 340-353. [CrossRef]

13. Ma, T.; Yan, B. The multiplicity solutions for nonlinear fractional differential equations of Riemann-Liouville type. Fract. Calc. Appl. Anal. 2018, 21, 801-818. [CrossRef]

14. Wang, Y.; Liu, Y.; Cui, Y. Infinitely many solutions for impulsive fractional boundary value problem with p-Laplacian. Bound. Value. Probl. 2018, 2018, 94. [CrossRef]

15. Zhang, L.; Zhou, Z. Spectral Galerkin approximation of optimal control problem governed by Riesz fractional differential equation. Appl. Math. Comput. 2019, 143, 247-262. [CrossRef]

16. Zhao, D.; Liu, Y.; Li, X. Controllability for a class of semilinear fractional evolution systems via resolvent operators. Commun. Pure Appl. Anal. 2019, 18, 455-478. [CrossRef]

17. Zhou, Z.; Gong, W. Finite element approximation of optimal control problems governed by time fractional diffusion equation. Comput. Math. Appl. 2016, 71, 301-318. [CrossRef]

18. Liu, Y.; Ahmad, B.; Agarwal, R.P. Existence of solutions for a coupled system of nonlinear fractional differential equations with fractional boundary conditions on the half-line. Adv. Differ. Equ. 2013, 2013, 46. [CrossRef]

19. Leung, A. A semilinear reaction-diffusion prey-predator system with nonlinear coupled boundary conditions: Equilibrium and stability. Indiana Univ. Math. J. 1982, 31, 223-241. [CrossRef]

20. Zhao, D.; Liu, Y. Positive solutions for a class of fractional differential coupled system with integral boundary value conditions. J. Nonlinear Sci. Appl. 2016, 9, 2922-2942. [CrossRef] 
21. Zhao, D.; Liu, Y. Eigenvalues of a class of singular boundary value problems of impulsive differential equations in Banach spaces. J. Funct. Space. 2014, 2014, 720494. [CrossRef]

22. Liu, Y. Existence and unboundedness of positive solutions for singular boundary value problems on half-line. Appl. Math. Comput. 2003, 144, 543-556. [CrossRef]

23. Yang, W. Positive solutions for nonlinear semipositone fractional q-difference system with coupled integral boundary conditions. Appl. Math. Comput. 2014, 244, 702-725. [CrossRef]

24. Asif, N.A.; Khan, R.A. Positive solutions to singular system with four-point coupled boundary conditions. J. Math. Anal. Appl. 2012, 386, 848-861. [CrossRef]

25. Cui, Y.; Liu, L.; Zhang, X. Uniqueness and existence of positive solutions for singular differential systems with coupled integral boundary value problems. Abstr. Appl. Anal. 2013, 2013, 340487. [CrossRef]

26. Cui, Y.; Zou, Y. An existence and uniqueness theorem for a second order nonlinear system with coupled integral boundary value conditions. Appl. Math. Comput. 2015, 256, 438-444. [CrossRef]

27. Yuan, C.; Jiang, D.; Regan, D.O.; Agarwal, R.P. Multiple positive solutions to systems of nonlinear semipositone fractional differential equations with coupled boundary conditions Electron. J. Qual. Theory Differ. Equ. 2012, 13, 1-17.

28. Zhao, D.; Liu, Y. Twin Solutions to Semipositone boundary value problems for fractional differential equations with coupled integral boundary conditions. J. Nonlinear Sci. Appl. 2017, 10, 3544-3565. [CrossRef]

29. Zhao, Q.; Yang, W. Positive solutions for singular coupled integral boundary value problems of nonlinear higher-order fractional q-difference equations. Adv. Differ. Equ. 2015, 2015, 290. [CrossRef]

30. Lakshmikantham, V.; Vatsala, A.S. Basic theory of fractional differential equations. Nonlinear Anal. 2008, 69, 2677-2682. [CrossRef]

31. Guo, D.; Lakshmikantham, V. Nonlinear Problem in Abstract Cones; Academic Press: San Diego, CA, USA, 1988.

32. Zhao, Y.; Sun, S.; Han, Z.; Li, Q. The existence of multiple positive solutions for boundary value problems of nonlinear fractional differential equations. Commun. Nonlinear Sci. Numer. Simulat. 2011, 16, 2086-2097. [CrossRef]

33. Tudorache, A.; Luca, R. Positive solutions for a system of Riemann-Liouville fractional boundary value problems with p-Laplacian operators. Adv. Differ. Equ. 2020, 2020, 1-30. [CrossRef] 